\title{
Nicardipine induces apoptosis via IRE1 activation in the PC12 cells.
}

\author{
Ji-Hye Song ${ }^{1 \#}$, O-Yu Kwon ${ }^{1 \#, ~ K i s a n g ~ K w o n ~}{ }^{2}$, Ji-Young Choi ${ }^{3}$, Seung-Whan Kim ${ }^{4}$ \\ ${ }^{1}$ Department of Anatomy and Cell Biology, College of Medicine, Chungnam National University, Research Institute for \\ Medical Sciences, Daejeon, Korea \\ ${ }^{2}$ Department of Biomedical Laboratory Science, College of Health \& Welfare, Kyungwoon University, Gumi, Korea \\ ${ }^{3}$ Applied Entomology Division, National Academy of Agricultural Science, RDA, Wanju, Korea \\ ${ }^{4}$ Department of Emergency Medicine, College of Medicine, Chungnam National University, Daejeon, Korea \\ \#These authors contributed equally to this work.
}

\begin{abstract}
Although an endoplasmic reticulum (ER) induction by calcium-channel blocker has been reported, the mechanism of ER stress-mediated apoptosis by nicardipine is unknown. Here, we demonstrated induction of apoptosis by nicardipine in PC12 cells. It was accompanied by the formation of apoptotic bodies via activation of ER-stress sensor, inositol-requiring enzyme 1 (IRE1). IRE1 induces the downregulation of key anti-apoptotic Bcl-2 expression in apoptosis. However, the altered expression of ER chaperones was not observed following nicardipine treatment. The results suggest that insight into the molecular mechanisms underlying nicardipineinduced apoptosis may facilitate the reduction of side effects and active treatment.
\end{abstract}

Keywords: Nicardipine, Endoplasmic reticulum (ER) stress, Inositol-requiring enzyme 1 (IRE1), B-cell lymphoma 2 (Bcl2), Apoptosis.

Accepted on February 07, 2018

\section{Introduction}

Nicardipine is one of the calcium-channel blocking agents that is commonly used as an antihypertensive agent in the setting of intracerebral hemorrhage and subarachnoid hemorrhage (Figure 1A) [1,2]. Several studies suggested that production of an abundance of oxygen radicals is one of the triggers of hypertension [3]. It is also demonstrated that calcium-channel blocker induces endoplasmic reticulum (ER) stress by disturbance of ER-lumen calcium homeostasis, finally leading to apoptosis [4]. However, the mechanism by which nicardipine induces apoptosis through ER stress is not completely understood.

The ER is a cytoplasmic organelle that mediates the synthesis, folding, modification, and transport of secretory proteins [5]. Various cellular stressors that disrupt the ER function lead to accumulation of un-, mis-, malfolded proteins in the ER lumen. ER stress induces ER-stress adaptable signal called the unfolded protein response (UPR) to restore ER homeostasis via activation of ER chaperones, in which three types of ER stress sensors including inositol requiring enzyme 1 (IRE1), PKRlike ER kinase (PERK) and activating transcription factor 6 (ATF6), are downstream ER chaperones [6,7].
Here, to provide a clue to understand the mechanism of nicardipine-induced apoptosis through the ER stress, we studied the effect of nicardipine on the activation of ER stressmediated apoptosis. One of the worst outcomes in hypertension, that is necessary of calcium channel blocking agent, is intracranial bleeding of central nervous system. Therefore, we used PC12 adrenal medulla pheochromocytoma cell line to help reduce the risk of bleeding in nerve cells or tissues.

\section{Materials and Methods}

PC12 cells were cultured in collagencoated flasks containing 85\% RPMI-1640 medium, supplemented with 25 mM HEPES buffer, $10 \%$ heatinactivated horse serum, 5\% heatinactivated fetal bovine serum, $2 \mathrm{mM}$ Lglutamine, $1 \mathrm{mM}$ sodium pyruvate, $1 \mathrm{~g} / \mathrm{l} \mathrm{d}(+)$ glucose, $25 \mu \mathrm{g} / \mathrm{ml}$ streptomycin and $25 \mathrm{U} / \mathrm{ml}$ penicillin (all Gibco; Thermo Fisher Scientific, Inc., Waltham, MA, USA). The cells were maintained in a humidified incubator at $37^{\circ} \mathrm{C}$ at $5 \% \mathrm{CO}_{2}$ and the medium was changed every $2 \mathrm{~d}$ [8]. At the beginning of this study, we tested the effects of nicardipine on cell viability in the MTT assay. Growth and viability of PC12 cells were determined using an MTT kit (SigmaAldrich). Color development was monitored at $595 \mathrm{~nm}$ with a reference wavelength of $650 \mathrm{~nm}$ using the 
Sunrise $^{\mathrm{TM}}$ microplate reader (Tecan Trading AG, Männedorf, Switzerland). As shown in Figure 1B, no obvious cell death was detected until $20 \mu \mathrm{g} / \mathrm{ml}$ nicardipine treatment and no remarkable morphological differences between nicardipinetreated and control cells were observed. Doses higher than $30 \mu \mathrm{g} / \mathrm{ml}$ nicardipine induced cytotoxicity. Each gene expression was mainly determined by RT-PCR as described below. RTPCR conditions included 30 cycles comprising each of the following: $94^{\circ} \mathrm{C}$ for $30 \mathrm{~s}, 58^{\circ} \mathrm{C}$ for $30 \mathrm{~s}$ and $72^{\circ} \mathrm{C}$ for $1 \mathrm{~min}$ (10 $\mathrm{min}$ in the final cycle) using the primers with Taq DNA polymerase (Solgent Co., Ltd. Daejeon, Korea).

Table 1. The gene names, GeneBank numbers and sequence of the primers used in the RT-PCR experiments and the reference gene PPIA.

\begin{tabular}{|c|c|c|}
\hline Gene & NCBI GenBank no. & Primer sequence \\
\hline \multirow{2}{*}{$P D I$} & \multirow{2}{*}{ X02918.1 } & F5'-CCA AAG CTG CTG CAA AAC TG-3' \\
\hline & & R5'-AAA GAG GAC CAC CCC ATC CT-3' \\
\hline \multirow{2}{*}{ BiP } & \multirow{2}{*}{ M14050.1 } & F5'-AGT GGT GGC CAC TAA TGG AG-3' \\
\hline & & R5'-TCT TTT GTC AGG GGT CGT TC-3' \\
\hline \multirow{2}{*}{ ERp29 } & \multirow{2}{*}{ BC091129.1 } & F5'-GGC ATC TTC ATC CCA GTC AT-3' \\
\hline & & R5'-TCC TCT GCA GCT CTT CCT TC-3' \\
\hline \multirow{2}{*}{ Calnexin } & \multirow{2}{*}{ NM_172008.2 } & F5'-GGC ATC TTC ATC CCA GTC AT-3' \\
\hline & & R5'-CTC CTC TCT GCT CCT CAT GG-3' \\
\hline \multirow{2}{*}{ PERK } & \multirow{2}{*}{ NM_031599.2 } & F5'-GGT CTG GTT CCT TGG TTT CA-3' \\
\hline & & R5'-TTC GCT GGC TGT GTA ACT TG-3' \\
\hline \multirow{2}{*}{ IRE1 } & \multirow{2}{*}{ NM_001191926.1 } & F5'-ACC ACC AGT CCA TCG CCA TT-3' \\
\hline & & R5'-CCA CCC TGG ACG GAA GTT TG-3' \\
\hline \multirow{2}{*}{ ATF6 } & \multirow{2}{*}{ BC168890.1 } & F5'-CTA GGC CTG GAG GCC AGG TT-3' \\
\hline & & R5'-ACC CTG GAG TAT GCG GGT TT-3' \\
\hline \multirow{2}{*}{ Bax } & \multirow{2}{*}{ NM_017059.2 } & F5'-AGG GGC CTT TTT GTT ACA GG-3' \\
\hline & & R5'-GAT CAG CTC GGG CAC TTT AG-3' \\
\hline \multirow{2}{*}{$B c / 2$} & \multirow{2}{*}{ L14680.1 } & F5'-AAG CTG TCA CAG AGG GGC TA-3' \\
\hline & & R5'-CAG ATG CCG GTT CAG GTA CT-3' \\
\hline \multirow{2}{*}{$X B P 1$} & \multirow{2}{*}{ NM_001004210.2 } & F5'-TCC TCC TGG GTA GAC CTC TG-3' \\
\hline & & R5'-AAA CAG AGT AGC AGC TCA GA-3' \\
\hline \multirow{2}{*}{ PPIA } & \multirow{2}{*}{ BC059141.1 } & F5'-GGT GAC TTC ACA CGC CAT AA-3' \\
\hline & & R5'-CTT CCC AAA GACCAC ATG CT-3' \\
\hline
\end{tabular}

The RTPCR primers were supplied by Bioneer Corporation (Daejeon, Korea). All chemicals were purchased from SigmaAldrich; Merck KGaA. The RTPCR primers used in this study are listed in Table 1.

The figures indicate the results of a representative experiment in triplicate with different samples.

\section{Results and Discussion}

We tested the role of nicardipine in inducing the expression of ER chaperones [9]. Protein disulfide isomerase (PDI) is also located in the ER lumen and catalyzes the formation and breakage of disulfide bonds between cysteine residues within proteins. Binding immunoglobulin protein $(\mathrm{BiP})$ is one of the ER molecular chaperones located in the ER lumen that binds newly-synthesized proteins during translation and, under normal conditions, is bound to three ER stress sensors. ER protein 29 (ERp29) shows sequence similarity to the PDI. It mediates the processing of secretory proteins within the ER. Calnexin is located in the ER membrane and facilitates quality control of unfolded or unassembled N-linked glycoproteins in the ER. As showed in Figure 1C, the expression of ER chaperones is not altered by any dose-dependent changes in nicardipine.


Figure 1. Effect of nicardipine on both cell viability and gene expression of ER chaperones. (A) The molecular structure of nicardipine (chemical formula, C26H29N3O6; molar mass, 479.525 $\mathrm{g} / \mathrm{mol})$; (B) PC12 cells were treated with $(1,5,10$ and $20 \mu \mathrm{g} / \mathrm{mL})$ nicardipine for $24 \mathrm{~h}$. Cell viability was measured by MTT assay. Cell viability was evaluated by EZ-cytox. The experiments were performed in triplicate and the results represent an average; (C) PC12 cells were treated with nicardipine (10 and $20 \mu \mathrm{g} / \mathrm{mL})$, tunicamycin and thapsicargin for $24 \mathrm{~h}$. Gene expression of ER chaperones was demonstrated by RT-PCR. The experiments were performed in triplicate and the results represent an average. Tu, tumicamycin; Thap, thapsicargin; PDI, protein disulfide isomerase; BiP, binding immunoglobulin protein; ERp29, endoplasmic reticulum protein 29.

Later, under similar experimental conditions as listed in Figure 1, we determined the expression of ER stress sensors (PERK, IRE1 and ATF6) (Figure 2A) [3]. Under altered ER homeostasis, the ER stress signal transduction pathway is mediated via activation of ER stress sensors. Although the expression of both PERK and ATF6 showed no remarkable changes following nicardipine treatment, the expression of IRE1 increased up to 3-fold. The stimulation of nicardipine appeared to selectively target the expression of IRE1 for UPR, rather than actively enhance the expression of ER stress chaperones. To further elucidate the unique IRE1 expression following nicardipine treatment, we tested the role of XBP1 mRNA splicing, just down-stream of IRE1 activation on the UPR. IRE1 activates the endonuclease domains, which cleave Xbox DNAbinding protein (XBP) mRNA, generating an 
activated form of the XBP1 mRNA for XBP1 protein [10]. We observed a dose-dependent increase in the spliced form XBP1 by nicardipine, indicated by a long arrow in Figure $2 \mathrm{~B}$. This result suggested that nicardipine only stimulates IRE1 mediated-XBP1 mRNA signaling, but not via PERK or ATF6, in the UPR.


Figure 2. Effect of nicardipine on the gene expression of ER stress sensors and XBP1 mRNA splicing. (A) PC12 cells were treated with nicardipine (10 and $20 \mu \mathrm{g} / \mathrm{mL}$ ), tunicamycin and thapsicargin for 24 $h$. The gene expression of ER stress sensors (PERK, IRE1 and ATF6) was confirmed by RT-PCR. The experiments were performed in triplicate and the results represent an average; (B) Nicardipine induces XBP1 mRNA splicing. The RT-PCR analysis was performed using $m R N A$ s listed in (A). The resulting PCR product was further digested by PstI to reveal a restriction site that was lost following $X B P 1$ splicing under ER stress. The resulting XBP1 cDNA products were revealed on a $2 \%$ agarose gel. Unspliced XBP1 $m R N A$ is indicated by a bold arrow. Tu, tumicamycin; Thap, thapsicargin; PERK, double-stranded RNA-activated protein kinase-like ER kinase; IRE1, inositol-requiring enzyme 1; ATF6, activating transcription factor 6 .

Here, we investigated the role of IRE1-XBP1 signaling in apoptosis induction. We performed two experiments to determine whether nicardipine induces chromatin condensation and apoptosis-associated gene expression, which are hallmarks of apoptosis [11]. We found that anti-apoptotic B-cell lymphoma $2(\mathrm{Bcl} 2)$ inhibits apoptotic death primarily by controlling the activation of caspase proteases. The proapoptotic $\mathrm{Bcl} 2$-associated $\mathrm{X}(\mathrm{Bax})$ is also a member of the $\mathrm{Bcl} 2$ family that promotes apoptosis. Eventually, the $\mathrm{Bax} / \mathrm{Bcl} 2$ ratio is one of the most important factors in determining apoptosis [12]. In this study, while nicardipine treatment decreased Bcl-2 expression, Bax expression remained unchanged (Figure 3A) suggesting that inhibition of Bcl-2 expression was an important step in nicardipine-induced apoptosis rather than the regulation of Bax expression. Next, different nuclei were observed in cells treated with and without nicardipine following Hoechst 33342 staining (Figure 3B). These results indicated that nicardipine was directly associated with induction of apoptosis in PC12 cells.

In summary, the present study for the first time demonstrated that nicardipine induces apoptosis via activation of IRE1-Bax pathway alone and not via activation of ER chaperones (PDI, BiP, ERp29 and calnexin) and ER stress sensors (ATF6 and PERK). Our findings suggest that nicardipine-induced PC12

cell apoptosis was associated with both ER sensors of IRE1 activation and down-regulation of Bax. It provides new insights into the possible role of nicardipine, and may help to attenuate its side effects when used in the treatment of vascular disorders such as chronic stable angina, hypertension, and Raynaud's phenomenon.



B


Figure 3. Nicardipine induces apoptosis in PC12 cells. (A) Effect of nicardipine on the gene expression of Bax and Bcl2-related apoptosis. The PC12 cells were exposed with nicardipine (10 and 20 $\mu \mathrm{g} / \mathrm{mL}$ ), tunicamycin and thapsicargin for $24 \mathrm{~h}$, respectively. Gene expression was indicated by RT-PCR. The experiments were performed in triplicate and the results represent an average; $(B)$ The cells were treated with nicardipine (10 and $20 \mu \mathrm{g} / \mathrm{mL}$ ) for $24 \mathrm{~h}$ and stained with Hoechst 33342 solution to detect the formation of apoptotic bodies indicated by arrows. Stained nuclei were observed under a fluorescent microscope using a blue filter. Tu, tumicamycin; Thap, thapsicargin; Bax, Bcl2 associated X; Bcl2, B-cell lymphoma 2.

\section{Acknowledgment}

This research was supported by Basic Science Research Program through the National Research Foundation of Korea funded by the Ministry of Education (NRF-2015R1D1A1A01057890).

\section{Conflict of Interest}

The authors have declared that no competing interests exist.

\section{References}

1. Dianne SS, Michael DB. The endoplasmic reticulum: structure, function and response to cellular signaling. Cell Mol Life Sci 2016; 73: 79-94.

2. Frakes AE, Dillin A. The UPRER: Sensor and coordinator of organismal homeostasis. Mol Cell 2017; 66: 761-771.

3. Back SH, Kaufman. RJ. Endoplasmic reticulum stress and type 2 diabetes. Annu Rev Biochem 2012; 81: 767-793.

4. Meister S, Frey B, Lang VR, Gaipl US, Schett G. Calcium channel blocker verapamil enhances endoplasmic reticulum stress and cell death induced by proteasome inhibition in myeloma cells. Neoplasia 2010; 12: 550-561. 
5. Pepine C. Nicardipine, a new calcium channel blocker: role for vascular selectivity. Clin Cardiol 1989; 12: 240-246.

6. Ortega-Gutierrez S, Thomas J, Reccius A, Agarwal S, Lantigua H, Li M, Carpenter AM. Effectiveness and safety of nicardipine and labetalol infusion for blood pressure management in patients with intracerebral and subarachnoid hemorrhage. Neurocrit Care 2013; 18: 13-19.

7. Montezano AC, Touyz RM. Molecular mechanisms of hypertensionreactive oxygen species and antioxidants: a basic science update for the clinician. Can J Cardiol 2012; 28: 288-295.

8. Kim SW, Yu K, Shin KS, Kwon K, Hwang TS, Kwon OY. Low-dose radiation suppresses Pokemon expression under hypoxic conditions. Z Naturforsch C 2014; 69: 68-74.

9. Garcia-Huerta P, Bargsted L, Rivas A, Matus S, Vidal RL. ER chaperones in neurodegenerative disease: Folding and $b$ eyond. Brain Res 2016; 1648: 580-587.

10. Hong DY, Kwon K, Lee KR, Choi YJ, Goo TW, Yu K, Kim SW, Kwon OY. Lidocaine induces endoplasmic reticulum stress-associated apoptosis in vitro and in vivo. Int $\mathrm{J}$ Mol Sci 2011; 12: 7652-7661.

11. Elmore S. Apoptosis: a review of programmed cell death. Toxicol Pathol 2007; 35: 495-516.

12. Wang Q, Zhang L, Yuan X, Ou Y, Zhu X, Cheng Z, Zhang P. The relationship between the Bcl-2/Bax proteins and the mitochondria-mediated apoptosis pathway in the differentiation of adiposederived stromal cells into neurons. PLoS One 2016; 11: e0163327.

\section{*Correspondence to}

Seung-Whan Kim

Department of Emergency Medicine

College of Medicine

Chungnam National University

Daejeon

Korea 